\title{
Correspondence
}

\section{Exploring ultrashort high-energy electron-induced damage in human carcinoma cells}

\author{
O Rigaud ${ }^{1}$, NO Fortunel ${ }^{1}$, P Vaigot ${ }^{1}$, E Cadio ${ }^{1}$, MT Martin ${ }^{1}$, O Lundh ${ }^{2}$, J Faure ${ }^{2}$, C Rechatin ${ }^{2}$, V Malka ${ }^{2}$ and YA Gauduel ${ }^{\star 2}$
}

Cell Death and Disease (2010) 1, e73; doi:10.1038/cddis.2010.46; published online 9 September 2010

Subject Category: Experimental Medicine

\section{Dear Editor,}

In conventional cancer therapy or fundamental radiobiology research, the accumulated knowledge on the complex responses of healthy or diseased cells to ionizing radiation is generally obtained with low-dose rates. Under these radiation conditions, the time spent for energy deposition is very long compared with the dynamics of early molecular and cellular responses. The energy depositions occur concomitantly with primary radio-chemical events (radical reactions), multiple biomolecular damage (membrane and DNA lesions), and repair. ${ }^{1}$ Such interferences may significantly influence the efficiency of signalling channels and repair processes or the recruitment of transducer proteins for programmed cell death or senescence. They render more complex or uncertain (i) a precise understanding of time-dependent relationships between the initial ionization density profile and the integrated complex response of normal or malignant tumour cells, and (ii) a complete description of biomolecule modifications and genome alterations resulting from energy deposition.

The use of ultrashort pulsed radiation would offer new perspectives for exploring the 'black box' aspects of long irradiation profiles and favouring the selective control of early damage in living targets. Several attempts were previously performed using nanosecond or picosecond pulsed irradiations on various mammalian cells and radiosensitive mutants at high dose rate. ${ }^{2-5}$ The effects of single or multi-pulsed radiations on cell populations were generally analyzed in the framework of dose survival curves or characterized by $2 \mathrm{D}$ imaging of $\gamma-\mathrm{H} 2 \mathrm{AX}$ foci and no increase in cytotoxicity was shown compared with a delivery at a conventional dose rate. Moreover, when multi-shot irradiations were performed, the overall time needed to obtain an integrated dose of several Grays again overlapped with the multi-scale dynamics of biomolecular damage-repair sequences and cell signalling steps.

Ideally, a single-shot irradiation delivering a well-defined energy profile, via a very short temporal window, would permit the approach of a real-time investigation of early radiationinduced molecular damage within the confined spaces of cell compartments. Owing to the potential applications of intense ultrashort laser for radiation therapy, ${ }^{6}$ the model of the A431 carcinoma cell line was chosen. An ultrafast single-shot irradiation strategy was carried out with these radio-resistant human skin carcinoma cells, ${ }^{7}$ using the capacity of an innovating laser-plasma accelerator to generate quasi mono-energetic femtosecond electron bunches in the $\mathrm{MeV}$ domain and to deliver a very high dose rate of $10^{13} \mathrm{~Gy} \mathrm{~s}^{-1}$ per pulse. ${ }^{8,9}$ The alkaline comet assay, ${ }^{10,11}$ which is commonly used to quantify global DNA damage in individual cells (single-, double-strand breaks, and alkali-labile sites), was applied to detect the impact of the $100 \mathrm{fs}$ single-shot $1 \mathrm{~Gy}$ exposure with electrons of mean energy $95 \mathrm{MeV}$ (Figure 1). The initial distribution of irradiated cells as a function of the comet tail moment, which reflects the level of DNA damage, shows a shift towards a population of more damaged cells, as compared with the sham-irradiated cells. The fraction of cells with damage above a control tail moment value of 4 exhibited an eightfold increase over that of the control cells. When carcinoma cells were maintained for $60 \mathrm{~min}$ at $37^{\circ} \mathrm{C}$ before the comet assay to allow DNA repair, the distributions of irradiated and non-irradiated cells became similar, indicating repair of the DNA lesions. The recovery of a near homogeneous distribution of low comet tail moments argues for the reparability of the global DNA lesions triggered by a single femtosecond irradiation shot at $1 \mathrm{~Gy}$. To assess the consequences of ultrafast electron-induced damage, the cytotoxicity was characterized in the same experiment, using a novel survival assay at a single-cell level. ${ }^{12}$ Two weeks after the femtosecond 1 Gy irradiation, a $95 \%$ survival was observed from a panel of 300 cells seeded in clonal microcultures.

This first investigation of a single-shot $1 \mathrm{~Gy}$ irradiation performed at high energy level and very high dose rate demonstrates that a measurable assessment of immediate and reversible DNA damage in carcinoma cells can be explored at the single-cell level. This breakthrough opens the possibility of a complete characterization of induced damage and repair, and notably of DNA double-strand breaks. In the framework of advanced spatio-temporal radiation biology concepts, ${ }^{13}$ one challenge of such a non-conventional irradiation concerns the complete understanding of the

\footnotetext{
'Laboratoire de Génomique et Radiobiologie de la Kératinopoïèse, CEA, 2 rue G. Crémieux, Evry 91057, France and 2LOA, CNRS UMR 7639, Ecole Polytechnique Paris Tech, ENSTA Paris Tech, Palaiseau Cedex 91761, France

*Corresponding author: Dr YA Gauduel, LOA, CNRS UMR 7639, Ecole Polytechnique Paris Tech, ENSTA Paris Tech, Palaiseau Cedex 91761, France.

Tel: + 331693197 26; Fax: + 331693199 96; E-mail: yann.gauduel@ensta-paristech.fr
} 
a

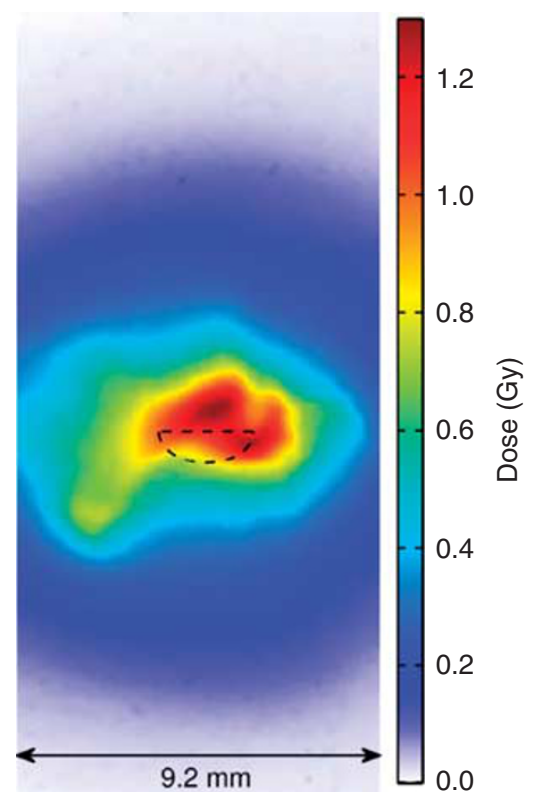

b

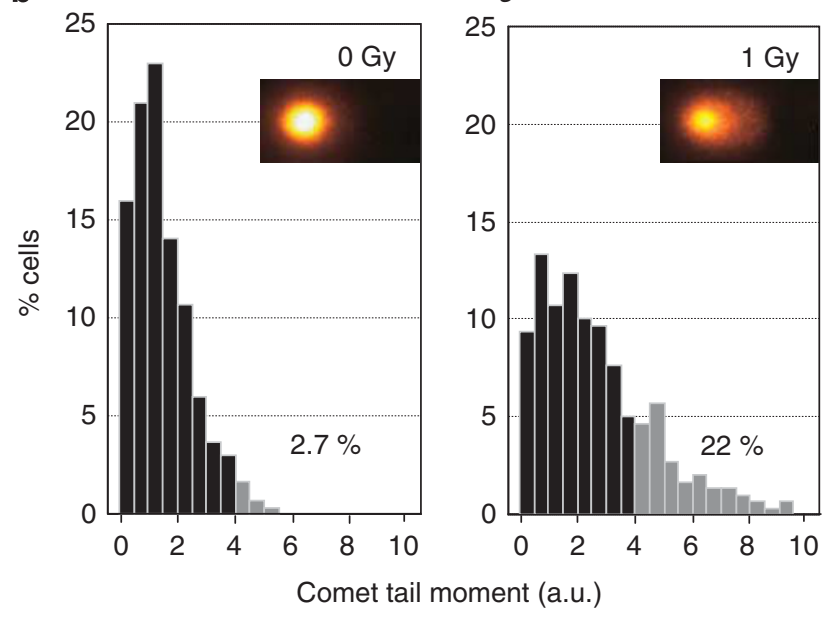

c

Residual DNA damage after repair

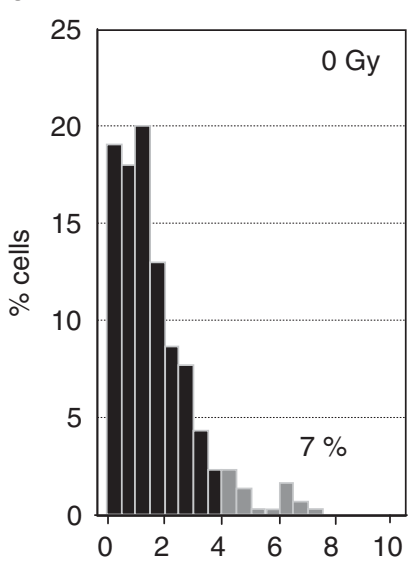

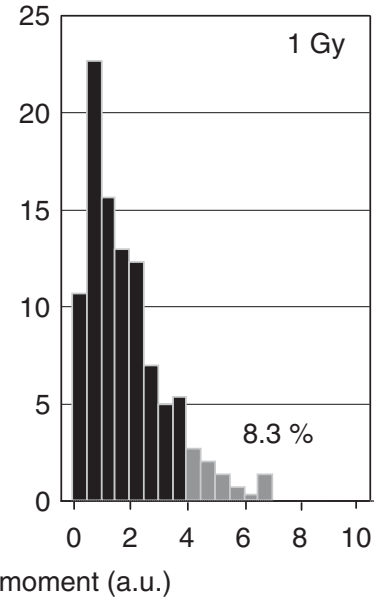

Figure 1 Induction of DNA damage in skin carcinoma cells irradiated at a very high dose rate by a single ultrashort bunch of high-energy electrons. (a) Image showing the overlap between the $2 \mathrm{D}$ dose deposition of a femtosecond quasi-monoenergetic electron bunch ( $100 \mathrm{fs}$ pulse duration, mean energy of $95 \mathrm{MeV}$ ) and the pellet containing about $5 \times 10^{5}$ human skin carcinoma cells. The dose delivered in the pellet (semi-ellipse) by an ultrafast single shot $\left(1.02 \pm 0.13 \mathrm{~Gy}\right.$, dose rate $10^{13}$ Gy s $\left.{ }^{-1}\right)$ is determined from Monte Carlo calculations. The exact number of electrons is deduced from scintillator measurements, which have been absolutely calibrated. ${ }^{9}(\mathbf{b}, \mathbf{c})$ Time-dependent evaluation of DNA damage using the alkaline comet assay for sham-irradiated ( 0 Gy) and irradiated carcinoma cells (1 Gy) immediately after the femtosecond irradiation (b) and after $1 \mathrm{~h}$ of repair time at $37^{\circ} \mathrm{C}$ (c). The frequencies of cells as a function of their damage level expressed by the comet tail moments are shown ( 300 comets analyzed per sample using Komet 6 software, Kinetic Imaging Ltd., Bromborough, UK). Gray bars represent the $\%$ of cells above a tail moment of 4 (arbitrary unit). A significant difference between the distributions of 0 and 1 Gy samples was found for initial damage $\left(\chi^{2}\right.$ test, $\left.P<0.002\right)$

multi-scale events triggered by the initial energy deposition, starting from the production and amplification of the localized radical processes and the induction of the primary lesions. A second challenge is deciphering the integrated cell response to these primary events, including cell signalling, damage sensing and DNA repair, and characterizing their late effects in cells, such as cell death, gene mutation and genomic instability. The emergence of ultrafast high-energy radiation biology could foreshadow the time-dependent and nanometric spatially defined effects in biomolecular architectures, such as aqueous groove of DNA, nucleosomes, protein pockets and sub-cellular compartments. Establishing an innovating approach of real-time nanodosimetry represents a prerequisite for the control of irradiations of living cells at very high dose rates. Moreover, the influence of the quality of shortpulsed particle beams (electrons and protons) on the relative biological efficiency (RBE) needs to be carefully evaluated, in synergy with ultrafast dose-fractionating protocols. Such knowledge is a necessary step before medical applications of ultrashort laser-accelerated particle beams. Currently, $\mathrm{X}$-rays in the few $\mathrm{MeV}$ energy range represent the majority of ionizing radiations used for cancer therapy. The dose deposited by very high-energy electron beams in the tissue depth is higher and could be beneficial to target deep 
tumors. ${ }^{14}$ Specific conditions afforded by very high dose rates and ultrashort dose fractionations would permit the real-time control of amplified radio-sensitivity during selective targeting protocols, in combination with the modern prodrug strategies developed in chemotherapy. ${ }^{15}$ Ultrafast radiation biology thus represents a newly emerging interdisciplinary field, in strong synergy with the most recent progresses of ultrashort radiation sources, high-energy bioradical femtochemistry, molecular biology and anti-cancer therapy.

\section{Conflict of interest}

The authors declare no conflict of interest.

Acknowledgements. We acknowledge the support of the European Research Council for funding the PARIS ERC project (contract number 226424). We also thank the support of the Comité de Radioprotection d'Electricité de France (EDF), Agence Nationale de la Recherche (contract number 08-CESA-024-04) and Radiation Biology MELUSYN Network (France).

\author{
1. Feuerhahn S, Egly JM. Trends Genet 2008; 24: 467-474 \\ 2. Tillman C et al. Radiology 1999; 213: 860-865. \\ 3. Shinohara K et al. J Radiat Res 2004; 45: 509-514. \\ 4. Kong X et al. Nucleic Acids Res 2009; 37: 2-14. \\ 5. Yogo A et al. Appl Phys Lett 2010; 94: 181502. \\ 6. Malka V et al. Nat Phys 2008; 4: 447-453. \\ 7. Pekkola-Heino K et al. Cancer Res 1989; 49: 4876-4878. \\ 8. Faure J et al. Nature 2006; 444: 737-739. \\ 9. Glinec $Y$ et al. Rev Sci Instrum 2006; 77: 103301 5-6. \\ 10. Olive PL, Banath J. Nat Protocols 2006; 1: 23-29. \\ 11. Gault N et al. Radiat Res 2007; 167: 551-562. \\ 12. Fortunel NO et al. Exp Dermatol 2010; 19: 387-392. \\ 13. Lacombe $\mathrm{S}$ et al. Cell Death Disease 2010; 1 : e4. \\ 14. Malka V, Faure J, Gauduel YA. Mutation Res Rev 2010; 704: 142-151. \\ 15. Kratz F et al. ChemMedChem 2008; 3: 20-53.
}

(c) SOMERIEHTS RESERVED

Cell Death and Disease is an open-access journal published by Nature Publishing Group. This work is licensed under the Creative Commons Attribution-Noncommercial-No Derivative Works 3.0 Unported License. To view a copy of this license, visit http://creativecommons.org/licenses/by-nc-nd/3.0/ 